\title{
Memoria de trabajo en consumidores de marihuana: estudio comparativo
}

Work memory in marijuana users: comparative study

Recibido el 30 de octubre del 2017, aceptado el 30 de noviembre del 2017

\begin{abstract}
*González, Valeria Alejandra
valeriagonzalez92@outlook.com

***Mustaca, Alba Elisabeth albamustaca@gmail.com

*Centro del Altos Estudios en Ciencias Humanas y de la Salud (CAECIHS) - Argentina

**Facultad de Psicología y HumanidadesUniversidad Abierta Interamericana (UAI) - Argentina
\end{abstract}

Para referenciar este artículo: Gonzálesz, V.A. y Mustaca, A.E. (2017) Memoria de trabajo en consumidores de marihuana: estudio comparativo. ConCiencia EPG, 2(2), 28-35.

\section{Resumen}

$\mathrm{E}$ 1 consumo de drogas ilícitas como la marihuana está aumentando de manera exponencial. Se demostró en el laboratorio que el consumo de estas sustancias provoca déficit cognitivos y emocionales. Sin embargo, no se hallaron estudios sobre el efecto de la marihuana en la memoria de trabajo (MT) con consumidores con una frecuencia media de consumo. Ante ello, el objetivo de este estudio fue, comparar la capacidad de la MT en consumidores de marihuana con aquellos que no lo son en 50 varones de 20 a 25 años. Los instrumentos utilizados en este estudio fueron, el Sub test de retención de dígitos de la escala de Wechsler de inteligencia para adultos y un cuestionario sociodemográfico general para recabar información sobre el inicio, forma y cantidad de consumo de marihuana. Los resultados evidencian que los no consumidores mostraron mayores puntuaciones en la MT la retención de dígitos hacia adelante, hacia atrás y en el puntaje total $(\mathrm{p}<0,05)$, aunque el tamaño del efecto fue mediano.

Palabras clave: marihuana, memoria inmediata, prueba de dígitos.

\section{Summary}

The consumption of illicit drugs like marijuana is increasing exponentially. It was demonstrated in the laboratory that the consumption of these substances causes cognitive and emotional deficits. However, no studies were found on the effect of marijuana on working memory (MT) with consumers with an average frequency of consumption. In view of this, the objective of this study was to compare the capacity of MT in marijuana users with those 
that are not in 50 men aged 20 to 25 years. The instruments used in this study were the Wechsler scale adult digit retention sub test for adults and a general socio-demographic questionnaire to gather information about the onset, form and amount of marijuana use. The results show that the non-consumers showed higher scores in the MT the retention of digits forward, backward and in the total score $(p<0.05)$, although the size of the effect was medium.

Keywords: marijuana, immediate memory, digit test

\section{Introducción}

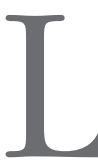

as drogas ilícitas aumentan su consumo a nivel mundial. Según datos estadísticos del año 2011, de los países miembros de la OEA, la más consumida en América es la marihuana. Entre el 2,9 y 4,3\% de la población de 15 a 65 años, ha consumido marihuana alguna vez. Su consumo más alto se observa entre los estudiantes de secundaria de Canadá (24\%) y los Estados Unidos (23\%), mientras que las más bajas están en Perú (1,9\%), Honduras (1\%), República Dominicana (1\%), Venezuela $(0,9 \%)$ y Haití $(0,7 \%)$. Su prevalencia asciende conforme aumenta la edad de los estudiantes del nivel secundario, lo cual es consistente en todos los países de los hemisferios americanos (CICAD, 2011).

En Argentina se hizo un estudio epidemiológico sobre la prevalencia y características del consumo de sustancias psicoactivas en una muestra representativa de 12.589 sujetos de 12 a 65 años (Cadenas \& Barberis, 2010). Según esa investigación, el consumo de marihuana se caracteriza por ser ocasional en el $54,7 \%$ de la población, frecuente, en el $37,7 \%$ y experimental, en el $6,8 \%$. En los varones tiene mayor peso el uso frecuente y cae el experimental y, a la inversa, en las mujeres, entre los adolescentes, casi un 21\% informa un uso frecuente, y en los mayores de 25 años, supera el 43\%. En cuanto a la modalidad de consumo, en localidades más pequeñas el peso del consumo es frecuente y bastante superior al de las de mayor tamaño ( $40,6 \%$ y $25,5 \%$ respectivamente). En estas últimas, el 18,5\% de los 69 consumidores recientes lo han hecho en forma experimental, mientras que, en áreas de menor población, esa modalidad de uso corresponde a menos del $4 \%$ de los usuarios del último año. El porcentaje de personas que son consumidoras de marihuana en los últimos 12 meses que presentan signos y síntomas de dependencia son casi el 18\%, unos 105.554 individuos. De cada 100 usuarios varones, 20 presentan problemas de uso compulsivo, tolerancia y síntomas de abstinencia, en tanto que, entre las mujeres, la relación fue menor. Según los grupos de edad analizados, aproximadamente 20 de cada 100 consumidores de 25 a 34 años presentan estos indicadores.

En Perú un estudio realizado con 4095 estudiantes de nivel secundaria, hallaron que el 9\% alguna vez consumió marihuana, en el último año, el 7,9\% y el último mes, el 5,9\%. El 24,1\% de los encuestados comenzó a consumir a los 15 años, y al igual que en otros países, la prevalencia fue mayor en los varones que en las mujeres (Bueno, Guerrero, Pedrajas \& Tam Phun, 2015). Estos datos muestran que la juventud es una de las poblaciones de mayor riesgo en el consumo de esta droga y que se deben evaluarse sus efectos y difundirlos en ámbitos educativos para alertar a los jóvenes sobre sus riesgos potenciales.

Aunque existen algunas controversias, hay trabajos que muestran que el consumo de marihuana provoca alteraciones cognitivas y emocionales. Una de las entidades con mayor renombre en el estudio de las drogas es el Instituto Nacional sobre el Abuso de Drogas que considera que el uso frecuente de marihuana deteriora la habilidad para formar memorias, recordar eventos, y para desviar la atención de una cosa a otra. Al mismo tiempo, el Tetrahidro cannabinol (THC), la droga más activa del cannabis, entorpece la coordinación y el equilibro al adherirse a los receptores en el cerebelo y a los ganglios basales, que son las partes del cerebro que regulan el equilibrio, la postura, la coordinación del movimiento y el tiempo de reacción (Volkow, 
2005). Por estos efectos, la intoxicación con marihuana puede provocar accidentes graves que pueden que terminar en la muerte del consumidor y en otros si son de tránsito.

Respecto de los estudios sobre los efectos del cannabis sobre la memoria, actualmente se obtuvieron resultados claros controlando posibles factores de confusión. En experimentos farmacológicos se investigaron los efectos agudos del delta-9-tetrahidrocannabinol ( $\triangle 9$-THC) y del cannabidiol (CBD), los ingredientes principales en el extracto de la planta de cannabis. Se hallaron resultados consistentes con deterioros agudos inducidos por una dosis única de $\triangle 9$-THC en la memoria verbal y de trabajo, sin quedar muy claro que persistan más allá del estado de intoxicación. A largo plazo, estas deficiencias parecen mantenerse y persistir después de la abstinencia si el uso regular y fuerte de las variedades de cannabis con un alto contenido de $\Delta 9$-THC se inicia a una edad temprana. Los estudios que emplearon técnicas avanzadas de neuroimagen comenzaron evaluar las bases neuronales de los efectos del consumo de cannabis hallando que hay una red de alteraciones funcionales y morfológicas que pueden moderar los efectos del cannabis en la función de la memoria (Schoeler \& Bhattacharyya, 2013).

Con humanos se halló un estudio realizado sobre el deterioro de la memoria de trabajo (MT) en adictos a drogas. La MT se refiere a la capacidad para mantener información un tiempo como para realizar acciones secuenciada. Está relacionada con la memoria de corto plazo, que es la capacidad para mantener la información durante segundos o minutos una vez pasado el momento que se recibe, tiene una capacidad limitada que se estima como la posibilidad de retener cinco o seis palabras, o siete dígitos (e.g., Sarubbo, 2013). Vo, Schacht, Mintzer \& Fishman (2015) evaluaron la memoria de trabajo (MT) en jóvenes consumidores primarios de opioides y de marihuana que ingresaron a una clínica de recuperación. Hallaron que después de 1 a 2 semanas de abstinencia y tratamiento, ambos grupos mostraron un deterioro de la MT con el uso de la Escala de Inteligencia de Wechsler.

De la bibliografía consultada, no se encontraron trabajos que evalúen la memoria de trabajo en jóvenes consumidores de uso poco o relativamente frecuente de marihuana y que realicen actividades propias de su edad, como estudiar y trabajar sin que se sientan afectados por su consumo. Estos estudios son importantes, ya que nos daría información sobre el deterioro de la MT en los sujetos que, de acuerdo a las investigaciones epidemiológicas, son los de mayor porcentaje en las poblaciones estudiadas. La siguiente investigación busca llenar comparar la MT en sujetos consumidores de uso frecuente de marihuana con aquellos que no consumen drogas con edades, niveles educativos y actividades similares. Se trata de un estudio expost-facto preliminar, con un diseño intersujeto con una variable independiente con dos valores (grupo consumidor de marihuana vs. no consumidor) y una variable dependiente (MT). De acuerdo a la bibliografía, se hipotetizó que los sujetos que consumen marihuana presentarán un déficit en el funcionamiento de la memoria de trabajo y que los que consumen con más frecuencia y que comenzaron más tempranamente a consumir presentarán mayor deterioro respecto de los demás. Los resultados buscan realizar una validación ecológica de los resultados obtenidos en el laboratorio y en adictos a la marihuana.

\section{Método}

Participantes: Se tomó una muestra seleccionada por conveniencia. Participaron voluntariamente 50 varones de entre 20 a 25 años, quienes se dividieron en dos grupos. 1) Consumidores de marihuana (GM, n= 25). Sujetos que consumían marihuana desde hacía al menos más de tres años $(n=25)$ y 2$)$ no consumidores (GnoM, n=25) sujetos que nunca consumieron marihuana ni otras drogas ilegales. En ambos grupos se excluyeron aquellos que tenían enfermedades psiquiátricas o psicológicas.

Instrumentos: El instrumento utilizado para medir la MT fue la escala de inteligencia para 
adultos de Wechsler- Tercera edición (WAIS III), versión renovada del test psicométrico desarrollado por David Wechsler (1999), que mantiene sus características fundamentales. La prueba incluye los 11 subtests del WAIS y tres nuevos: "Razonamiento con matrices", "búsqueda de símbolos" y "Ordenamiento de númerosletras". Se halla compuesto por una escala verbal y una escala de ejecución. Para esta investigación se utilizó solamente el subtest de dígitos de la escala verbal, que evalúa la MT, la que se considera un tipo especial de memoria cuya capacidad consta de la retención de elementos durante un tiempo determinado que permite realizar acciones secuenciadas (Torres \& Fiestas, 2012).

El subtest de dígitos consta de dos partes: retención de dígitos hacia adelante y retención de dígitos hacia atrás, siendo la retención de dígitos hacia adelante la primera en administrarse. La prueba consiste en presentar al sujeto una serie de dígitos, que debe repetir de forma inmediata, en orden serial y en voz alta. Cada nivel se define por la cantidad de dígitos a recordar entre 2 y 8 en dígitos hacia adelante, y entre 2 y 7 dígitos hacia atrás y se presentan dos series por nivel. Al comienzo se dan a conocer las instrucciones y luego se le presenta al individuo una serie de dos dígitos hacia adelante y se van agregando el número de dígitos a recordar. La prueba se discontinúa cuando el sujeto no logra recordar correctamente las dos series que componen el nivel. Finalizada la administración de dígitos hacia adelante, al sujeto se le presenta una serie de dígitos, que debe repetir en el orden serial inverso. Cada nivel tiene una cantidad de dígitos a recordar (entre 2 y 7) que se despliegan de a dos series por nivel. La puntuación se realiza en base a las reglas de interrupción, y teniendo en cuenta las puntuaciones correspondientes a la cantidad de dígitos hacia adelante y hacia atrás que pudo recordar, y al puntaje total entre ambos dígitos, de puntuación desarrolladas en la escala de inteligencia para adultos de Wechsler- Tercera edición (1999).
El cuestionario sociodemográfico fue utilizado para recoger información del lugar de residencia, estado civil, oficio u ocupación, estudios cursados, consumo de marihuana, edad de su inicio, frecuencia y última vez que consumió.

Procedimiento: Cada participante fue evaluado individualmente. Se les informaba sobre los objetivos de la investigación y luego de la firma del consentimiento se les administraba primero el cuestionario sociodemográfico y por último la prueba. Las pruebas se administraron aproximadamente a la misma hora (5-7 p.m.) y con el GM se constataba que no hubieran consumido marihuana dentro de las 5 horas anteriores a la administración de la prueba con el fin de controlar los efectos agudos de su consumo.

Análisis de datos: Se utilizó el programa SPSS. Se estableció como criterio de significación, alfa $<0,05$ y el tamaño del efecto de acuerdo a la fórmula de $r=Z$ /raíz cuadrada $N$. Los valores del tamaño del efecto se consideran: $r=<0,1$, efecto nulo:, $r$ entre $>0,1$ a $<0,3$ : efecto pequeño; $r$ entre $>0,3$ a $<0,5$,efecto mediano; y $r>0,5$, efecto grande.

\section{Resultados}

Caracterización de la muestra: La edad de los sujetos de toda la muestra se distribuyen de la siguiente manera: el $12 \%(\mathrm{n}=6)$, tenía 20 años; el $24 \%(n=12), 21$ años; el 24\% $(n=12), 22$ años; el 20\%( $n=10), 23$ años; $16 \%(n=8), 24$ años y el $4 \%(n=2), 25$, años. En cuanto al estado civil, el $82 \%(n=41)$ era soltero y el $18 \%(n=10)(n=41)$, el $16 \%$ se encontraba en pareja $(n=9$ era casado o vivía con su pareja. El $44 \%(n=22)$ era empleado, el $52 \%$, $(n=26)$, y el $4 \%(n=2)$ es desempleado. En cuanto al nivel educativo, el 64\% tenían nivel universitario completo $(n=13)$ o incompleto $(n=$ 19), el $22 \%$, terciario incompleto $(n=7)$ o terciario completo $(n=4)$, y el $14 \%$ poseía secundario incompleto $(n=4)$ o secundario completo $(n=3)$.

El GM era el 52\% (n=13) empleado, el 40\% $(n=10)$, estudiante el $4 \%(n=1)$, desempleado, y el $4 \%(n=1)$, otras actividades. En cuanto al nivel de 
estudios, el $12 \%(n=5)$ poseía secundario completo o incompleto, el $32 \%$, terciario incompleto $(n=8)$, terciario completo o incompleto) y el $65 \%(n=14)$, universitario incompleto $(\mathrm{n}=13)$ o completo $(\mathrm{n}=1)$. En cuanto a la frecuencia de consumo, el $36 \%(n=9)$, consumía una vez por semana, el $40 \%$ $(n=10)$, de dos a tres veces por semana y el $24 \%$ $(n=6)$ más de tres veces por semana. Respecto a la cantidad de cigarrillos que fumaba en cada episodio, el $60 \%(n=15)$, consumía de uno a tres cigarrillos, el 32\% (n=8) consumía de 4 a 5 y el $8 \%$ $(n=2)$, más de 5 . En cuanto al último momento de consumo, el 8\% lo hizo el mismo día de la prueba, $(n=2)$, el 14\% ( $n=7)$, el día anterior, el 28\% $(n=14)$ la semana anterior y el $4 \%(n=2)$, el mes anterior. Los dos sujetos que consumieron el mismo día de la administración fue durante la mañana (entre las 9-10 a.m.), por lo cual no se descartaron de la muestra. Un participante manifestó consumir otra sustancia ilegal por lo cual fue descartado del análisis estadístico.

Prueba de Normalidad: Los resultados de la prueba Kolmogórov-Smirnov mostraron que dígitos para atrás y puntaje total en el GM no cumplían con los criterios de normalidad, por lo cual se utilizaron medidas no paramétricas para las pruebas de inferencias (Ver Tabla 1).

\section{Tabla 1}

\section{Prueba de normalidad}

\begin{tabular}{|c|c|c|c|c|}
\hline \multicolumn{2}{|c|}{ Var./Consume marihuana } & \multicolumn{3}{|c|}{ Kolmogórov-Smirnov } \\
\hline & & Estadístico & $\mathrm{gl}$ & Sig.p $<$ \\
\hline D. hacia adelante & $\begin{array}{l}\mathrm{Si} \\
\quad \mathrm{No}\end{array}$ & $\begin{array}{l}0,116 \\
0,155\end{array}$ & $\begin{array}{l}25 \\
25\end{array}$ & $\begin{array}{l}0,200 \\
0,126\end{array}$ \\
\hline D. hacia atrás & $\begin{array}{l}\mathrm{Si} \\
\quad \mathrm{No}\end{array}$ & $\begin{array}{l}0,191 \\
0,150\end{array}$ & $\begin{array}{l}25 \\
25\end{array}$ & $\begin{array}{l}0,000 \\
0,151\end{array}$ \\
\hline P. Total & $\begin{array}{l}\mathrm{Si} \\
\quad \mathrm{No}\end{array}$ & $\begin{array}{l}0,189 \\
0,108\end{array}$ & $\begin{array}{l}25 \\
25\end{array}$ & $\begin{array}{l}0,021 \\
0,200\end{array}$ \\
\hline
\end{tabular}

\section{Memoria de trabajo en función de los grupos}

La Tabla 2 muestra el promedio y desvío estándar de la prueba de dígitos en función de los grupos. Se observa que en las tres pruebas los participantes que no consumieron marihuana tuvieron un puntaje mayor respecto de los que lo hicieron. Un análisis con la prueba U-Mann Whitney informa que estas diferencias son significativas, aunque el tamaño del efecto es pequeño: dígitos hacia adelante: $Z=-2,805(p<0,005), r=0,40$; dígitos hacia atrás: $Z=-2,780(p<0,005), r=0,39$; puntaje total: $Z=-3,199(p<0,005), r=0,45$. 
Tabla 2

Memoria de trabajo en función del consumo de maribuana

\begin{tabular}{|c|c|}
\hline Consume marihuana & $\mathrm{M}(\mathrm{DS})$ \\
\hline $\begin{array}{l}\text { D. hacia adelante Si } \\
\qquad \mathrm{No}\end{array}$ & $\begin{array}{l}7,44(1,80) \\
9,12(1,94)\end{array}$ \\
\hline $\begin{array}{l}\text { D. hacia atrás } \quad \text { Si } \\
\quad \mathrm{No}\end{array}$ & $\begin{array}{l}5,16(1,86) \\
6,56(2,00)\end{array}$ \\
\hline P. Total & $\begin{array}{l}12,60(2,66) \\
15,72(3,42)\end{array}$ \\
\hline No & \\
\hline
\end{tabular}

Grupo consumidores de marihuana

\section{MT y edad de comienzo de consumo}

En la tabla 3 muestra la MT en función de la edad del comienzo del consumo. Los resultados evidencian la presencia de diferencias grupales en función de la edad de comienzo de consumo de marihuana. La prueba de Kruskal-Wallis arroja que hay diferencias significativas solamente en dígitos hacia atrás (chi cuadrado 8,72, p < 0,014). Dado que son tres grupos, se procedió a analizar con la prueba U- Mann Whitney comparando grupos de pares para averiguar entre qué grupos se encuentra la significación estadística de dígitos hacia atrás.
Este análisis arrojó diferencias significativas entre los sujetos que comenzaron antes de los 14 años respecto de entre 18-20 años (chi cuadrado $=8,50$, $\mathrm{p}<0,01)$ y entre 14-17 años respecto de 18-20 años (Chi cuadrado $=7,48, \mathrm{p}<0.006$ ). Esto indica que en dígitos hacia atrás puntuaron significativamente menos los sujetos que comenzaron a consumir entre los 18-25 años respecto de los que lo hicieron desde los 14-17 y antes de los 14 años. El último resultado hay que tomarlo con precaución dado que el número de sujetos que comenzó antes de los 14 años es muy reducido (3 sujetos).

Tabla 3

Memoria de trabajo y comienzo del consumo de marihuana

\begin{tabular}{llll}
\hline Edad de consumo por primera vez & N & Rango promedio \\
D. hacia adelante & Antes de los 14 años & 3 & 10,00 \\
& Entre 14 y 17 años & 11 & 13,45 \\
& Entre 18 y 20 años & 11 & 13,36 \\
Total & 25 & \\
D. hacia atrás & Antes de los 14 años & 3 & 12,33 \\
& Entre 14 y 17 años & 11 & 17,50 \\
& Entre 18 y 20 años & 11 & 8,68 \\
P. total & Total & 25 & \\
& Antes de los 14 años & 3 & 10,00 \\
& Entre 14 y 17 años & 11 & 16,50 \\
& Entre 18 y 20 años & 11 & 10,32 \\
\hline
\end{tabular}

MT, frecuencia de consumo y número de cigarrillos por episodio 
El análisis de rangos promedios muestra que los sujetos que consumen más de tres veces por semana puntúan menos en dígitos hacia adelante que el resto. Sin embargo, los resultados no fueron significativos $(p>0,05)$. Lo mismo ocurre en cuanto al número de cigarrillos consumidos por episodio $\mathrm{p}>0.05$ ).

\section{Discusión y Conclusiones}

Los estudios realizados con animales de laboratorio y sujetos adictos que estaban en tratamiento hallaron deterioro en la MT (Farre \& Abanades, 2007; Monckerberg, 2014 , y Schacht, Mintzer \& Fishman,2015). Los resultados obtenidos en el presente trabajo se halló un deterioro de la MT, pero en personas consumidoras frecuentes no considerados adictos, y se los comparó con un grupo control homogéneo en edades, sexo y nivel socioeconómico y cultural.

En cuanto al grupo consumidor, los resultados arrojan diferencias significativas dependiendo de la edad de inicio de consumo de marihuana, pero no de acuerdo a la hipótesis planteada. En dígitos hacia atrás los consumidores que obtuvieron menor puntaje fueron aquellos que comenzaron a consumir entre los 18-25 años respecto de los que consumían desde los 14 a17 años y antes de los 14 años. Este último resultado, sin embargo, debe ser tomado con cautela ya que el grupo de que consumió antes de los 14 años fue reducido (3 sujetos) respecto de los otros dos (11 sujetos cada uno). Con respecto a la frecuencia de consumo, si bien se halló que aquellos que consumían más de tres veces por semana obtuvieron menor puntaje en dígitos hacia adelante, las diferencias no fueron significativas. En relación a la cantidad de cigarrillos consumidos tampoco se encontraron diferencias significativas, al igual que el estado civil, nivel de estudios y oficio u ocupación. Estos datos sugieren que aun con baja frecuencia de consumo ya puede haber algún déficit en la MT.
Lo más relevante a tener en cuenta, son los resultados arrojados de la comparación entre consumidores-no consumidores de marihuana, en la cual se hallaron diferencias significativas aunque el tamaño del efecto fue mediano. Es de hacer notar que, si bien los consumidores de marihuana obtuvieron puntajes menores que los no consumidores, los promedios de los dígitos recordados por ambos grupos de comparación están dentro de los límites que sugiere la teoría de la memoria de corto plazo que, respecto a su capacidad, está comúnmente aceptada la cifra promedio de $7 \pm 2$ elementos (Sarubbo, 2009). Es de destacar que el test de memoria de dígitos, relativamente sencillo de administrar, ya encuentra deterioro entre los consumidores no adictos lo que sugiere que el efecto de la marihuana sobre la MT es potente, aunque su tamaño del efecto sea mediano y probablemente imperceptible para la vida cotidiana de ese tipo de consumidores.

Dado que este trabajo es limitado en cuanto a número de sujetos evaluados, se destaca la importancia de seguir estudiando los posibles deterioros cognitivos y emocionales en consumidores no adictos de marihuana para evaluarlos con más detalle, aumentando la muestra en función de la frecuencia, cantidad y edad del consumo ya que, como se informó en la introducción, el consumo de drogas ilegales está aumentando en la población mundial y ya hay países que la han legalizado.

Con respecto a los alcances de esta investigación, sus resultados alcanzan una mayor validez ecológica a las investigaciones de laboratorio que conviene difundir, junto con otros datos sobre los efectos de la marihuana, para incorporarlos en los programas de prevención de drogas, y para la toma decisiones en la gestión pública acerca de su uso legal. 


\section{Referencias}

Bueno, L., Guerrero, J., Javier, R. \& Tam Phun, E. (2015). Prevalencia de consumo de marihuana en estudiantes de secundaria de instituciones educativas estatales de Ventanilla. Revista de enfermería Herediana, 8,17-23. doi:10.20453/ renh.v8i1.2537

Cadenas, N., \& Barberis, L. (2010). Estudio nacional en población de 12 a 65 años, sobre consumo de sustancias psicoactivas. Recuperado de http://www.observatorio.gov.ar/media/k2/ attachments/reZConsumoZdeZSustanciasZPsicoactivasZenZPoblacionZdeZ12ZaZ65ZAniosZAnioZ2010.pdf

CICAD (2011). Informe del uso de drogas en las Américas. Washington: OID. Recuperado de http://www.cicad.oas.org/oid/pubs/Uso_de_ Drogas_en_Americas2011_Esp.pdf

Farré, M., \& Abanades, S. (s.f.). Aspectos cognitivos del consumo de cannabis. En Aspectos psiquiátricos del consumo de cannabis. (pp. 93-105). Madrid: Sociedad Española de investigación en cannabinoides. Recuperado de http://www.biblioteca.cij.gob.mx/Archivos/ Materiales_de_consulta/Drogas_de_Abuso/ Articulos/Diagnostico1.pdf
Monckeberg, F. (2014). Los pro y contra de la legalización de la marihuana. Revista chilena de pediatría, 85(2), 229-231. Recuperado de http://www.scielo.cl/pdf/rcp/v85n2/art14.pdf

Schoeler, T. \& Bhattacharyya, S.(2013). The effect of cannabis use on memory function: an update. Substances Abuses and Rehabilitation, 4, 11-27. doi: 10.2147/SAR.S25869.

Vo, H. T., Schacht, R., Mintzer, M., Fishman, M. (2014). Working memory impairment in cannabis- and opioid-dependent adolescents. Substaces Abuses, 35(4), 387-390. doi: 10.1080/08897077.2014.954027

Volkow, N. (2005). Abuso de la marihuana. Serie de reportes (13-3859). Recuperado de http:// www.descentralizadrogas.gov.co/wp-content/uploads/2015/02/Serie-Reportes-Investigacion-Abuso-de-la-marihuana-NIDA.pdf

Wechsler, D. (1999). WAIS-III. Escala de inteligencia de Wechsler para adultos-III. Madrid: TEA (Edición original, 1997). 